\title{
El reto del trabajo interdisciplinar: ¿hasta dónde hemos llegado?
}

RESUMEN: Este trabajo se propone investigar como está distribuida la asunción de tareas asistenciales entre los profesionales de salud mental de la Comunidad de madrid, fundamentalmente psiquiatras y psicólogos. A la luz de estos datos, analiza hasta donde se refrenda y potencia el modelo comunitario de atención, supuestamente basado en la interdisciplinariedad y las responsabilidades compartidas.

PALABRAS CLAVE: Psicología clínica, salud mental comunitaria, interdisciplinariedad.
SUMMARY: The objetive of this article is to research how the clinical work )diagnosis, treatments, etc.) is distributed among the mental helath professionals of the Community of Madrid, mainly between psychiatrists and psychologists. The community mental health based on the interdisciplinary team is also analyzed.

KEY WORDS: Clinical Psychology, community mental helath, interdisciplinary work.

\section{Introducción.}

A partir de la promulgación de la Ley General de Sanidad se inicia oficialmente un proceso de reforma en materia de salud, a través de la creación del Sistema Nacional de Salud que incorpora las prestaciones y servicios de salud mental en el sistema general. La reforma es importante, no solo porque acaba con la tradicional marginación de este tipo de trastornos, sino porque concibe la salud mental como un campo interdisciplinario sujeto a diferentes disciplinas.

El Informe de la Comisión Ministerial para la Reforma Psiquiátrica concretaba el desarrollo de dichas prestaciones en el subsistema de salud mental, proponiendo un nuevo modelo y señalando además la necesidad de incluir nuevas profesiones y disciplinas. Este modelo descentralizaba la atención del hospital psiquiátrico y daba paso a una concepción de red de servicios diversificados de salud mental, sobre un área definida e integrada en el Sistema Nacional de Salud, como servicios especializados de segundo y tercer nivel. Establecía que las prestaciones debían estar basadas en una concepción interdisciplinaria y comunitaria, mencionando explícitamente la incorporación de los psicólogos clínicos en dicha red. En definitiva se proponía un modelo para entender el proceso salud-enfermedad desde unos parámetros diferentes al modelo biológico.

Los equipos encargados de realizar esta cobertura son equipos de composición multidisciplinaria. Es decir clínicos especializados en materia de salud mental, entre los que están psiquiatras y psicólogos, así como otros profesionales que realizan diversas funciones: trabajadores sociales, DUEs, terapeutas ocupacionales, etc.

Este modelo de atención supone necesariamente la coordinación y colaboración conjunta de todos los profesionales que forman parque del equipo especializado de salud mental. La calidad de la atención depende, por lo tanto, de que este modelo se aplique y 
lleve a la practica correctamente. Esto supone la realización de las tareas clínicas propias de cada disciplina, entre otros de los psiquiatras y psicólogos. Entre ellas están la evaluación y el diagnóstico clínico, los tratamientos y seguimientos de pacientes, las indicaciones de ingresos y cualquier actuación que se considere necesaria para la correcta atención del paciente.

Con el estancamiento de la reforma psiquiátrica aparecen diferentes conflictos, entre otros los que giran en torno a la realización y responsabilidad correspondiente de estas tareas. Implicando un riesgo de involución para todo el sistema de atención al poner en peligro la asistencia integral y las competencias y responsabilidades compartidas de la red de servicios territorializados, dejando de lado otros graves problemas que debemos abordar.

El objetivo de este trabajo, realizado desde la Asociación Madrileña, que agrupa a diferentes profesionales y cuyo interés es continuar trabajando por el sostenimiento de la calidad asistencial de los Servicios Públicos de S.M., es contribuir a la clarificación de las competencias y responsabilidades clínicas que son propias de una disciplina, en este caso la Psicología Clínica. Nos parece necesario separar dos cuestiones que, aunque estén relacionadas son esencialmente diferentes. Una cuestión es la legalidad vigente,que se funda en lo ya establecido y sólo se renueva con posterioridad a los cambios que se operan en la sociedad, y de la cual se derivan responsabilidades específicas para los especialistas (psiquiatras y psicólogos); y otra cuestión diferente son las competencias y responsabilidades clínicas propias de cada disciplina . Tanto la responsabilidad clínica como la legal, dependiendo de cada situación, deberían ser compartidas por cada una de las disciplinas que intervengan en el abordaje de cada caso, enfocando la cuestión como una corresponsabilidad dentro del equipo clínico.

El planteamiento de este estudio es señalar cómo la materialización del trabajo interdisciplinar, se da con ciertas confusiones e interdicciones en la práctica clínica, porque conviven dos niveles de funcionamiento: el real, en el que se da de hecho la corresponsabilidad, y el legal-institucional en el que para todo lo que respecta al paciente el responsable último, y casi único, es el psiquiatra.

\section{Estudio de las actividades asistenciales en la Comunidad de Madrid.}

Nos planteamos investigar cómo estaba distribuida la asunción de tareas asistenciales entre los profesionales de salud mental, especialmente psiquiatras y psicólogos, para posteriormente estudiar los datos obtenidos y extraer conclusiones en cuanto al tema que nos preocupa esto es, si existe una profundización cada vez mayor en la descentralización de las tareas y en la asunción de responsabilidades.

\section{Sujetos y Método}

Población

En el momento del estudio existen en Madrid (capital y provincia) 35 Servicios de Salud Mental de Distrito. El cuestionario sobre "Actividades Clínico-Asistenciales de los Facultativos" se envió por correo, dirigido personalmente a cada Jefe de Servicio, solicitando su colaboración para transmitir dicho cuestionario a los distintos facultativos 
ORIGINALES Y REVISIONES

del servicio, que deberían contestarlo individual o colectivamente y devolverlo en un plazo máximo de 20 días.

\section{Recogida de Datos}

Se diseñó un cuestionario de 20 ítems con respuesta múltiple, de las cuales diez correspondían al Programa de Adultos y otras diez al Programa Infanto-juvenil.

Los ítems se refieren a las distintas actividades clínico-asistenciales (10 para cada programa) que pueden desarrollar los distintos facultativos (Psiquiatras y Psicólogos, fundamentalmente), pero daban la opción de responder si dichas actividades eran desarrolladas por otros profesionales, solicitando fuese especificado por cuáles.

Alguno de los ítems del cuestionario dio lugar a una interpretación confusa, que comentaremos más detalladamente al hablar de los resultados.

El cuestionario podía ser respondido individual o colectivamente por cada equipo y no aportaba datos de caracterización sobre el sujeto que respondía (edad, sexo, categoría profesional...). En algunos equipos respondieron distintos profesionales de forma individual, siendo sus respuestas en ocasiones similares y en ocasiones contradictorias entre sí. Decidimos considerar las respuestas contradictorias como no excluyentes, y las respuestas similares como una sola respuesta por Servicio, de modo que agrupamos las respuestas de cada equipo como si hubieran respondido colectivamente a un único cuestionario que incluiría todas las respuestas, pero sin multiplicarlas.

Es obvio que los datos que recoge este cuestionario se refieren a la percepción subjetiva que tienen los profesionales sobre quién desarrolla las distintas actividades clínico asistenciales en su equipo de trabajo.

Se ha recogido información de los 35 distritos, aunque no todos ha aportado datos sobre ambos Programas. Sobre el Programa de adultos han respondido 33 distritos (el 94,2\%) y sobre el Programa Infanto-juvenil lo hicieron 31 distritos (el 88,6\%)

\section{Resultados}

Los resultados globales se presentan en las dos tablas adjuntas (Tablas 1 y 2), en las cuales se pueden ver el número de respuestas por categoria profesional (n) y el porcentaje (\%) que representa " $n$ " respecto al número de casos considerados válidos (los que dan en ese item al menos una respuesta en alguna de las categorías profesionales). profesional.

A continuación comentaremos los resultados obtenidos, analizados por categoría

En el Programa de Adultos, los psiquiatras desarrollan en mayor porcentaje que el resto de las categorías profesionales todas las actividades clínico-asistenciales evaluadas, excepto los tratamientos de psicoterapia $(69,7 \%)$. Los psicólogos desarrollan casi todas las actividades en menor porcentaje que los psiquiatras, excepto los tratamientos de psicoterapia (100\%).

Es de destacar que en un $22 \%$ de los casos (corresponde a 7 distritos) los psicólogos no realizan tareas de Evaluación y Diagnóstico. Estos distritos son: Torrejón en el area 3, Tetuán en el area 5, Chamberí y Latina en el area 7, Alcorcón, Navalcarnero y Móstoles en el area 8 y Arganzuela y Usera en el area 11. En el distrito de Móstoles los psicologos 
asumen tareas de diagnóstico pero con una selección previa del tipo de trastorno. Nos parece que este $22 \%$, que representa casi la cuarta parte de los distritos, en los que los psicólogos estan apartados de una de sus funciones específicas, es indicativo de una retirada de la responsabilidad clínica sobre los pacientes porque además se filtra a través del criterio de otro profesional qué pacientes son susceptibles de tratamiento psicológico. Es llamativo que, como veremos después, este filtro no es necesario en el caso de los programas infanto juveniles. Por otra parte, tambien es notable que este modo de distribución de las tareas de diagnóstico discrepe con lo plasmado en el Programa de Salud Mental de la Comunidad de Madrid, donde se especifica que tanto psiquiatras como psicólogos asumirán estas tareas.

En el Programa Infanto-juvenil, psiquiatras y psicólogos desarrollan al 50\% las distintas actividades. Los psicólogos realizan más entrevistas de evaluación y diagnóstico, tratamientos de psicoterapia, informes para la comisión de tutela, coordinación con atención primaria y coordinación con servicios educativos. Los psiquiatras realizan en mayor porcentaje tratamiento farmacológico, informes judiciales, informes para otros especialistas, indicación de ingresos hospitalarios y coordinación con dispositivos hospitalarios.

Respecto al personal de enfermería y a los trabajadores sociales, ambos participan fundamentalmente en las actividades de coordinación con los dispositivos sanitarios y educativos y, en el Programa Infanto-juvenil; el trabajador social también en la realización de informes para la Comisión de Tutela. En algunos casos también ambos realizan primeras entrevistas de evaluación.

Los médicos de Atención Primaria y los pediatras realizan tratamientos farmacológicos en el $12,5 \%$ de los casos en el programa de adultos, y en un $6,7 \%$ en el programa Infanto-juvenil.

Por último, señalar que en 7 casos $(21,2 \%)$ ningún profesional realiza Informes de IVE, y en otro $3(9,1 \%)$ ninguno prescribe bajas/altas laborales. En menos porcentaje también nos encontramos con que en algunos centros nadie realiza actividades de coordinación con dispositivos hospitalarios (en el caso del programa Infanto-juvenil, puede que no existan dichos dispositivos), ni con atención primaria.

A continuación, quisiéramos realizar algunos comentarios referidos a los ítems que constituyen el cuestionario:

- Primeras entrevistas de evaluación y diagnóstico.

Aunque el cuestionario solo daba la posibilidad de responder si dicha tarea la desarrollaba el Psiquiatra y/o el Psicólogo, en algunos casos se incluyó como respuesta: enfermera, trabajador social...

Ello nos hizo pensar que estábamos hablando de 2 tareas diferentes: la evaluación inicial, que efectivamente es desarrollada en algunos centros (probablemente en más de los que respondieron así) por personal de enfermería 
y/o trabajador social, y la entrevista de diagnóstico, que es desarrollada por psiquiatra y/o psicólogo. El ítem incluye las dos tareas (evaluación y diagnóstico) pero no dimos la opción de responder "Otros profesionales". Esta omisión ha sesgado los resultados, pues ha unificado dos tareas que en la práctica cotidiana de muchos centros no son realizadas sólo por psiquiatras o psicólogos.

Al analizar este ítem habremos de entenderlo, por tanto, como referido a entrevistas "con objetivos de diagnóstico" fundamentalmente.

- Informes de I.V.E.

Este ítem daba la opción de responder psiquiatra/psicólogo. Pensamos que esta doble opción indujo también a confusión pues, obviamente, los psicólogos no pueden firmar informes de IVE, ya que no tienen reconocida capacidad legal para ello. Sólo el psiquiatra la tiene.

Entendemos que los centros que han respondido que el psicólogo realizaba dicha actividad se refieren a la realización de informes clínicos que apoyan los informes firmados por el psiquiatra, o a la firma conjunta psiquiatra/psicólogo de dichos informes.

Algunos centros dejaron en blanco este ítem, y lo interpretamos como que en dicho centro ningún profesional realizaba esta actividad. De nuevo se pone de manifiesto la disociación entre los dos niveles de funcionamiento, ya que, siguiendo la letra del cuarto supuesto de la Ley de I.V.E., se trata de valorar el posible daño psicológico para la madre, aspecto que en la práctica evalúan tambien los psicólogos pero al que no pueden dar refrendo legal.

- Informes Judiciales.

En este ítem algunos centros contestaron también argumentando que el reconocimiento legal para firmar informes judiciales es exclusivamente del psiquiatra. En realidad, la ley no impide a los psicólogos firmar este tipo de informes, aunque la práctica cotidiana se desarrolla muchas veces bajo este "supuesto" que sólo es una determinada interpretación de la ley quizá demasiado rigurosa, o demasiado dependiente de "lo que se ha hecho siempre". De hecho, en el programa infantil, los datos reflejan que tanto los informes judiciales como los informes para la Comisión de Tutela, son firmados de manera equiparable por psiquiatras y psicólogos. ¿Cuál puede ser la razón por la que se nos reconoce capacidad legal para informar sobre la cuestión de si a una familia se le retira o no la custodia o tutela de un niño, y, sin embargo, no tendríamos capacidad legal para informar a un juez sobre otros aspectos que se refieren a nuestros pacientes.

- Prescripción de bajas/altas laborales.

Como en el caso de los informes de IVE, se daba la posibilidad de una doble respuesta psiquiatra/psicólogo. Obviamente, los psicólogos no pueden firmar 
bajas ni altas laborales, aunque, como muchos centros aclaran, sí recomiendan al médico de Atención Primaria dicha prescripción.

Una duda que nos surge a partir de este comentario es la de en cuántos centros los psiquiatras prescriben realmente, es decir firman Altas/bajas y en cuántos sólo recomiendan al médico de atención primaria.

Algunos centros dejaron el ítem en blanco y lo incluímos como "Ninguno", sumando dichas respuestas a las de los que sí contestaron aclarando explícitamente que ningún profesional realizaba dicha tarea, aunque ambos recomendaban.

- Indicación de Ingresos hospitalarios:

Aquí los datos revelan que esta actividad es asumida siempre por psiquiatras y que en un $32 \%$ de los casos no la realizan los psicólogos. Esto significa que o bien en un $32 \%$ de los distritos los psicólogos no ven nunca pacientes que necesiten ingresar o bien existe una inercia institucional que les excluye de la tarea misma. Así, en algunos dispositivos son los responsables mismos de estas unidades los que excluyen a los psicólogos en sus protocolos de derivación a las mismas. Y, aún más contundente en este sentido, es una reciente circular de la Jefa de Salud Mental, recordando que para ingresar en las unidades de hospitalización de nifos y adolescentes es siempre necesaria la firma de un psiquiatra. Nuestros datos dicen que hay distritos sin psiquiatra en el programa infanto-juvenil, y que aún así la indicación del ingreso la realiza este profesional....ंQué criterio es el que determina este sin sentido?

- Coordinación Hospitalaria y coordinación con atención primaria.

Por una parte diremos que las tareas de coordinación son las más compartidas por todos los profesionales del equipo básico, pero también es dónde se recogen más contradicciones en las respuestas. Existen distritos de los que hemos recibido varios cuestionarios con respuestas discrepantes en las actividades que tienen que ver con lo hospitalario.Los datos reflejan que los psiquiatras asumen mayoritariamente estas tareas, pero también DUES y trabajadores sociales. La discrepancia puede hacemos pensar también que, o bien no está claro quién realiza la tarea, o incluso, si la coordinación se entiende como un programa, como un contacto telefónico o incluso como una fantasía imaginaria. Por ejemplo, en distritos donde nos consta que no hay programa de niños,existen actividades asistenciales con niños; no hay psiquiatra infantil, pero la indicación de ingreso, los tratamientos farmacológicos, la coordinación con dispositivos hospitalarios la realiza el psiquiatra...(el de adultos, suponemos). Por último, hay que señalar que en algunos distritos en los que se respondió que no existe coordinación con dispositivos hospitalarios para el programa infanto juvenil, se debe a que no existen estos dispositivos. 


\begin{tabular}{|c|c|c|c|c|c|c|c|}
\hline \multirow{2}{*}{ 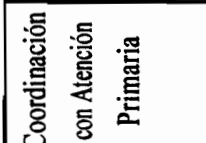 } & $\alpha^{\circ}$ & बे & $\frac{a}{\infty}$ & बे & & గీ & $m$ \\
\hline & $=$ & $\bar{m}$ & ๙ิ & $m$ & & 0 & - \\
\hline \multirow{2}{*}{ 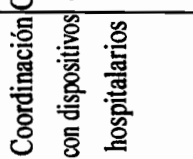 } & $\partial^{\circ}$ & $\frac{a}{\infty}$ & $\frac{n}{n}$ & 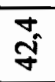 & & $\hat{m}_{m}^{m}$ & $m$ \\
\hline & $=$ & શิ & $\simeq$ & $\Xi$ & & $=$ & - \\
\hline \multirow{2}{*}{ 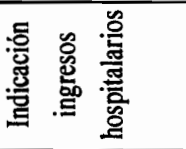 } & $\partial^{\theta}$ & 8 & $\begin{array}{l}0 \\
\hat{\sigma}\end{array}$ & & & & \\
\hline & $=$ & $\dot{m}$ & $\ddot{\sim}$ & & & & \\
\hline \multirow{2}{*}{ 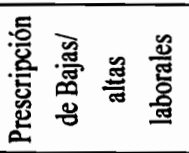 } & $\Delta^{0}$ & $\hat{\tilde{\Omega}}$ & $\hat{n}_{n}^{n}$ & & & & $\bar{a}$ \\
\hline & $=$ & 요 & $\stackrel{\circ}{\sim}$ & & & & $m$ \\
\hline \multirow{2}{*}{ 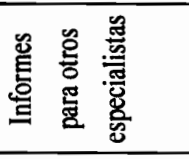 } & $0^{0}$ & 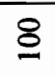 & बे & & & & \\
\hline & $=$ & $\hat{m}$ & ల్ & & & & \\
\hline \multirow{2}{*}{ 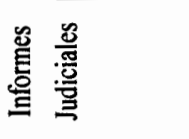 } & $\partial^{\circ}$ & 8 & $\begin{array}{l}\dot{0} \\
\tilde{n}\end{array}$ & & & & \\
\hline & $=$ & $\tilde{m}$ & $\underline{2}$ & & & & \\
\hline \multirow{2}{*}{ 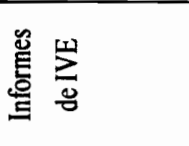 } & $a^{\circ}$ & $\stackrel{\infty}{\infty}$ & $\underset{\infty}{\infty}$ & & & & $\frac{N}{N}$ \\
\hline & $a$ & $\hat{\sim}$ & 0 & & & & r \\
\hline \multirow{2}{*}{ 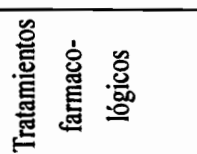 } & $\partial^{\circ}$ & 8 & & & $\cong$ & & \\
\hline & $=$ & N & & & $\nabla$ & & \\
\hline \multirow{2}{*}{ 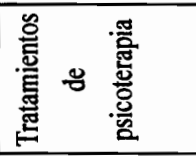 } & $\partial^{\circ}$ & $\hat{8}$ & 8 & & & & \\
\hline & $=$ & $\tilde{\sim}$ & $\tilde{m}$ & & & & \\
\hline \multirow{3}{*}{ 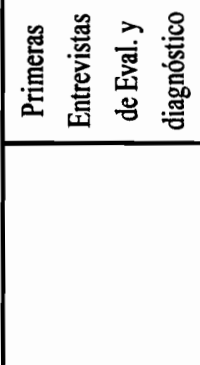 } & $d^{\circ}$ & 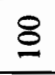 & $\infty$ & $m$ & & $m$ & \\
\hline & $=$ & $\tilde{m}$ & ా & - & & - & \\
\hline & 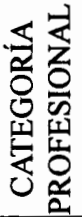 & $\begin{array}{l}\frac{5}{2} \\
\vdots \\
\vdots \\
\vdots \\
0\end{array}$ & 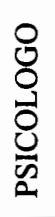 & 岇 & 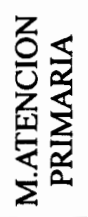 & 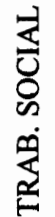 & $\begin{array}{l}O \\
Z \\
\text { Z } \\
z \\
Z\end{array}$ \\
\hline
\end{tabular}




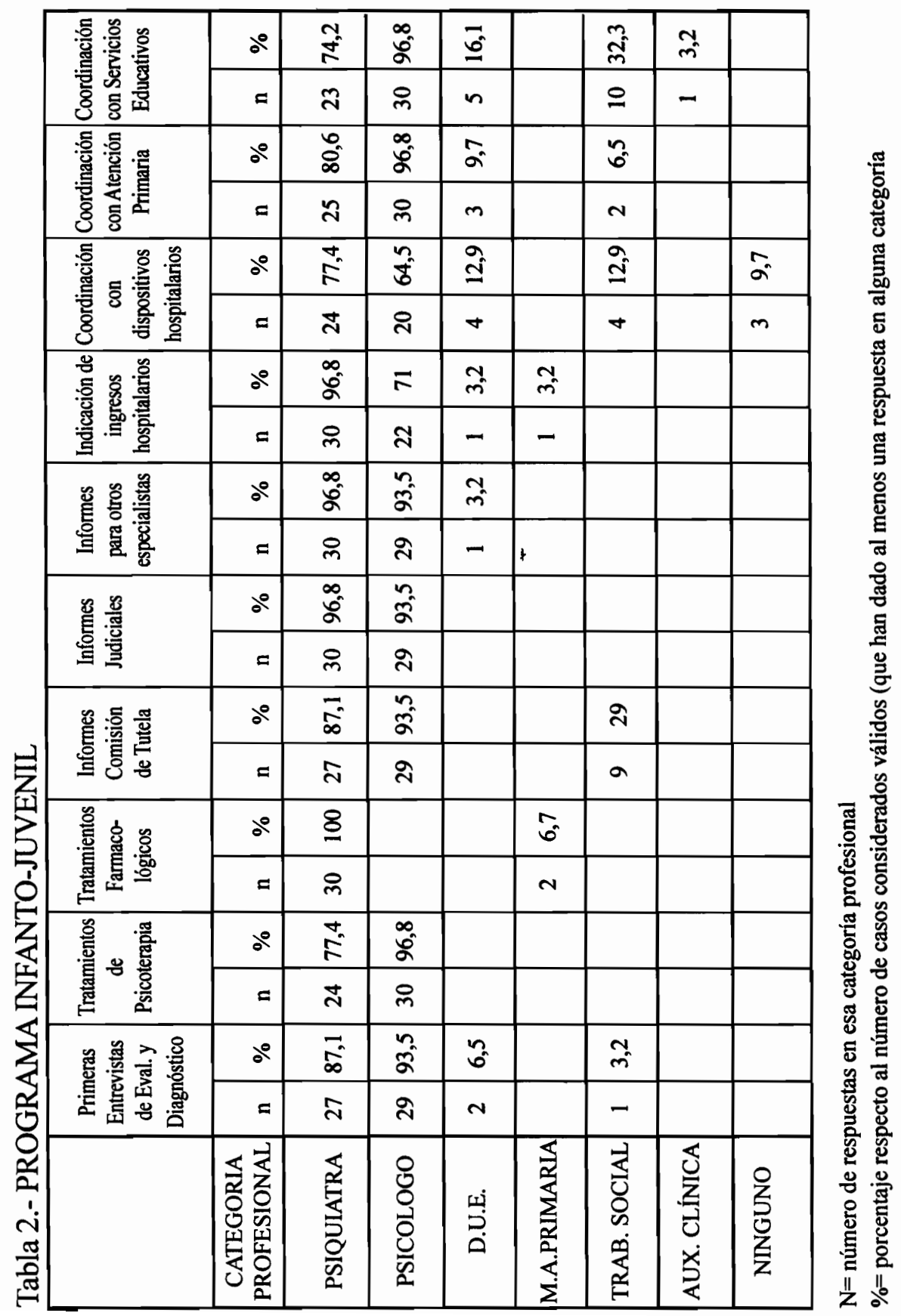




\section{ANALISIS COMPARATIVO}

\begin{tabular}{|c|c|c|c|c|c|c|}
\hline \multicolumn{2}{|c|}{} & $\begin{array}{c}\text { Entrevista } \\
\text { de } \\
\text { Evaluación } \\
\text { y } \\
\text { Diagnóstico }\end{array}$ & $\begin{array}{c}\text { Tratamiento } \\
\text { de } \\
\text { Psicoterapia }\end{array}$ & $\begin{array}{c}\text { Informes } \\
\text { Judiciales } \\
\text { Hospitalario }\end{array}$ & $\begin{array}{c}\text { Indicación } \\
\text { de } \\
\text { Ingreso } \\
\text { Hospitalarios }\end{array}$ & $\begin{array}{c}\text { Coordinación } \\
\text { con } \\
\text { Dispositivos }\end{array}$ \\
\hline \multirow{2}{*}{ Psiquiatra } & Adultos & $100 \%$ & $69,7 \%$ & $100 \%$ & $100 \%$ & $100 \%$ \\
\cline { 2 - 7 } & Infantil & $87,1 \%$ & $77,4 \%$ & $96,8 \%$ & $96,8 \%$ & $77,4 \%$ \\
\hline \multirow{2}{*}{ Psicólogo } & Adultos & $78 \%$ & $100 \%$ & $57,6 \%$ & $67,6 \%$ & $51,5 \%$ \\
\cline { 2 - 7 } & Infantil & $93,5 \%$ & $96,8 \%$ & $93,5 \%$ & $71 \%$ & $64,5 \%$ \\
\hline
\end{tabular}

Fecha de recepción: 16/7/01

* S.S.M. de Getafe, S.S.M. de Usera, S.S.M. de Getafe, S.S.M. de Barajas,

S.S.M. de Ciudad Lineal, S.S.M. de Navalcarnero, S.S.M. de Majadahonda, respectivamente

Correspondencia:

María Luz Ibáñez.

Servicio de S.M. de Getafe, Avda. de los Angeles, 53 - Getafe, 28903 Madrid 